\title{
All-Fiber Spatial Mode Selective Filter for Compensating Mode Dependent Loss in MDM Transmission Systems
}

\author{
Y. Jung, ${ }^{*}$ S.U. Alam, and D.J. Richardson \\ Optoelectronics Research Centre, University of Southampton, Southampton, SO17 1BJ, UK \\ *ymj@orc.soton.ac.uk
}

\begin{abstract}
We propose and demonstrate a simple all-fiber spatial mode selective filter based on splicing a short segment of small-core SMF between two few-mode fibers. An 8dB MDL equalization range with less than $2 \mathrm{~dB}$ IL is demonstrated for a 2-mode fiber device. OCIS codes: (060.0060) Fiber optics and optical communications; (060.2340) Fiber optics components.
\end{abstract}

\section{Introduction}

Space division multiplexing (SDM), in particular mode division multiplexing (MDM) [1-2], offers the potential to open up the spatial dimension as a means to increase the per-core transmission capacity, i.e. spectral efficiency (bits/s/Hz), in optical fiber communication systems. In a few-mode fiber (FMF) based MDM system, one of the critical issues is the mitigation of mode dependent loss (MDL) (or mode dependent gain (MDG)) which can lead to significant loss of overall performance (i.e. reduced capacity/transmission distances). Generally, higher-order modes (HOMs) in a FMF have a larger mode field diameter (and larger evanescent field) and typically suffer higher attenuation and coupling losses in FMF based optical components. Also, few-mode fiber amplifiers typically exhibit a preferential modal gain for the lower-order modes (LOMs) and both careful tailoring of the doping profile and pump spatial mode control have been employed to help mitigate MDG [3]. Consequently, in most MDM transmission systems HOMs experience greater optical losses than LOMs. In order to address this it is necessary to develop MDL compensation devices, in particular suitable LOM filters, to achieve optimum system performance. However, realization of an LOM filter is not simple because LOMs are very well confined within the core compared to HOMs. To address this issue, the first dynamic MDL equalization filter has been demonstrated at OFC' 14 which worked by blocking the central portion of the beam with a spatial light modulator [4]. The fundamental $\mathrm{LP}_{01}$ mode was selectively attenuated over a 0 to $10 \mathrm{~dB} \mathrm{MDL}$ equalization range, however the higher-order $\mathrm{LP}_{11}$ mode experienced an increasingly high insertion loss (IL) as the equalization range was increased (i.e. $5 \mathrm{~dB}$ and $15 \mathrm{~dB} \mathrm{IL}$ for 5 and 10dB MDL equalization respectively). Moreover, use of a spatial light modulator represents an expensive way to realize MDL compensation. An all-fiber approach would be preferable offering potential benefits in terms of low insertion loss, low cost and ready integration within fiber systems.

In this paper, we propose and demonstrate a simple all-fiber solution for spatial mode filtering achieved by splicing a short segment of small-core single-mode fiber (SMF) between two FMFs. The small-core SMF in the middle creates two spatial optical pathways (i.e. a guided mode along the core and a radiated diffracting beam within the fiber cladding). These beams serve to define a Mach-Zehnder interferometer (MZI) [5], which can be used to ensure that the LOMs experiences higher loss relative to HOMs through destructive interference as the light is coupled back into the second section of FMF. To prove the principle we investigated two mode group filters supporting $\mathrm{LP}_{01}$ and $\mathrm{LP}_{11}$ modes. An $8 \mathrm{~dB}$ MDL equalization range was successfully achieved providing a reasonable insertion loss $(<2 \mathrm{~dB})$ for the favored $\mathrm{LP}_{11}$ mode.

\section{FMF-SMF-SMF fiber structure for LOM filtering}

The proposed all-fiber spatial mode selective filter is schematically illustrated in Fig. 1 which, as described above, is in effect a compact Mach-Zehnder interferometer. When the input $\mathrm{LP}_{01}$ mode from $\mathrm{FMF} 1$ arrives at the small-core

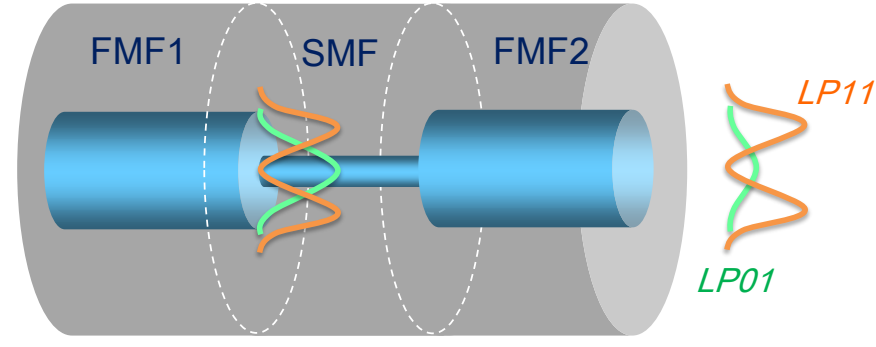

Fig. 1. Schematic of the FMF-SMF-FMF fiber structure for lower-order mode (LOM) filtering. 
SMF as shown in Fig. 2(a), a portion of the beam is launched into the central small-core region (whose dimensions and NA need to be carefully chosen) and the remnant annular portion gradually diffracts into the pure silica cladding. After accumulating an optical path length difference along the SMF, the two optical beams recombine at FMF2, where interference occurs which modulates the transmission response. The phase relationship between the two optical paths depends on the wavelength and the structural parameters of the small-core fiber (i.e. core diameter and NA). The total required phase difference can be calculated through the expression $\left(\delta \varphi=2 \pi \Delta n_{\text {eff }} L / \lambda\right)$, where $\Delta n_{\text {eff }}$ is the effective refractive index difference between the core and cladding modes of the small-core SMF and $L$ is the interferometer length. The HOMs on the other hand have a much smaller modal overlap with the central small core region and as a result experience less loss. To illustrate and validate the principle of operation we performed beam propagation method (BPM) simulations (using the commercial RSoft package) of a device based on a step-index two-mode fiber supporting $\mathrm{LP}_{01}$ and $\mathrm{LP}_{11}$ modes (core diameter $=20 \mu \mathrm{m}, \mathrm{NA}=0.12$ ). The results for a small-core $\mathrm{SMF}$ segment with a core diameter of $5 \mu \mathrm{m}$ and a numerical aperture (NA) of 0.17 are shown in Fig. 2. As depicted on the right side of the Fig. 2(a) the monitored optical power (i.e. coupled power into the $\mathrm{LP}_{01}$ mode of the second FMF) exhibits a sinusoidal character as the propagation length within the small-core is increased and reaches a first minimum (destructive interference $(\delta \varphi=\pi)$ ) around $L=140 \mu \mathrm{m}$. For $\mathrm{LP}_{11}$ mode propagation, the two-lobed LP 11 mode exhibits very little overlap with the core region of the small-core SMF and most of power simply diverges into the cladding, resulting in a monotonic decrease in the monitored power (i.e. coupled power into the $\mathrm{LP}_{11}$ mode of the second FMF) in Fig. 2(b). By examining the near field patterns along the beam propagation, the two beam interference effect of the proposed device can clearly be visualized (Fig. 2(c)). The Gaussian-shaped LP mode $_{01}$
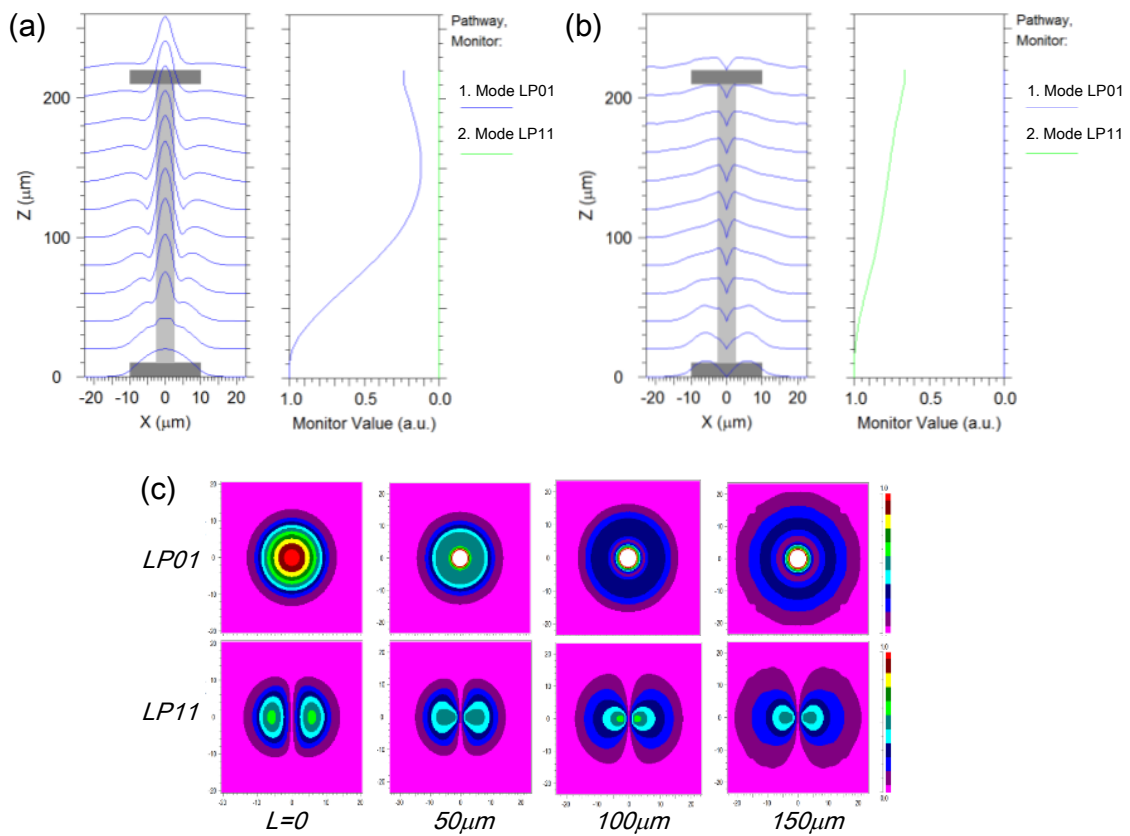

Fig. 2. BPM simulated optical field distribution through the FMF-SMF-FMF structure and calculated coupling loss for the pure (a) $\mathrm{LP}_{01}$ and (b) $\mathrm{LP}_{11}$ mode excitation. (c) Series of simulated near-field patterns obtained at different position of the small-core SMF along the optical pathway.
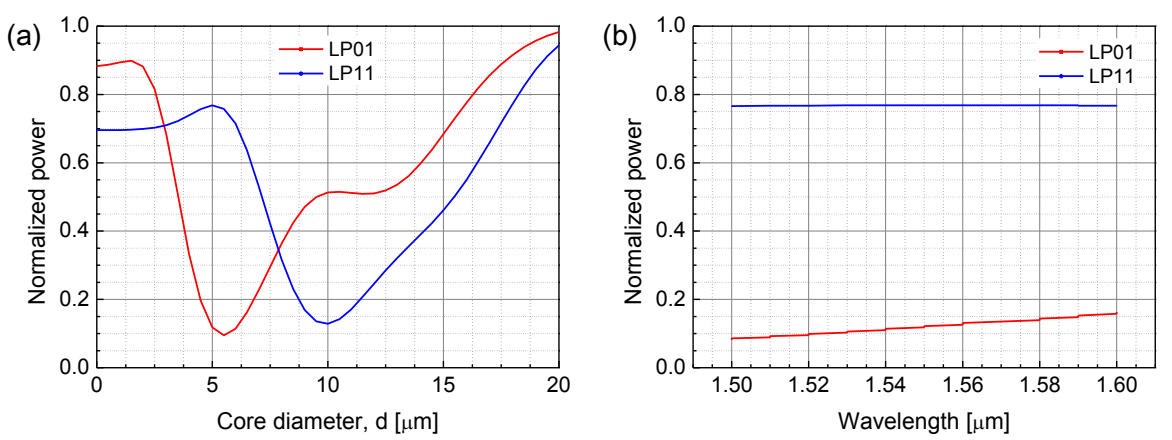

Fig. 3. (a) Spatial mode filtering dependence on the core diameter of the SMF; the optimum core diameter is $d=5.5 \mu \mathrm{m}$ for LOM filtering and $d=10 \mu \mathrm{m}$ for HOM filtering at $1550 \mathrm{~nm}$. (b) The wavelength dependence of the LOM filter at the optimized parameters $(d=5.5 \mu \mathrm{m}, L=140 \mu \mathrm{m})$. 
substantially overlaps with the central small-core and splits into two optical beams; a central Gaussian-shaped core guided beam and an outer ring-shaped diverging beam. A clear dark ring (no optical intensity) is observed between the two beams owing to the abrupt $\pi$-phase shift, showing that the small-core SMF acts like a binary phase plate with a central circular aperture. For the $\mathrm{LP}_{11}$ mode propagation, the two lobed $\mathrm{LP}_{11}$ beam experiences little perturbation from the central small-core and it gradually diffracts from the central axis into the cladding.

Fig. 3(a) shows the dependence of the mode filtering on the core diameter of the SMF as predicted by the BPM simulations. For small core diameters $(2.5-8 \mu \mathrm{m})$, the proposed device exhibits LOM filter behavior because the Gaussian-shaped $\mathrm{LP}_{01}$ mode suffers destructive interference due to the MZI effects. As the core diameter of the SMF is further increased $\left(>8 \mu \mathrm{m}\right.$ ), the $\mathrm{LP}_{11}$ mode undergoes greater attenuation than the $\mathrm{LP}_{01}$ mode due to the enhanced field overlap with the increased core, and the device exhibits HOM filtering behavior. The optimum core diameter, $d$, is $5.5 \mu \mathrm{m}$ for a LOM filter and $d=10 \mu \mathrm{m}$ for a HOM filter at $1550 \mathrm{~nm}$. Fig. 3(b) shows the wavelength dependence of the LOM filter at the optimized parameters $(d=5.5 \mu \mathrm{m}, L=140 \mu \mathrm{m})$. It shows a broadband flat response extending over the whole C-band because we choose the first destructive interference point of the MZI.
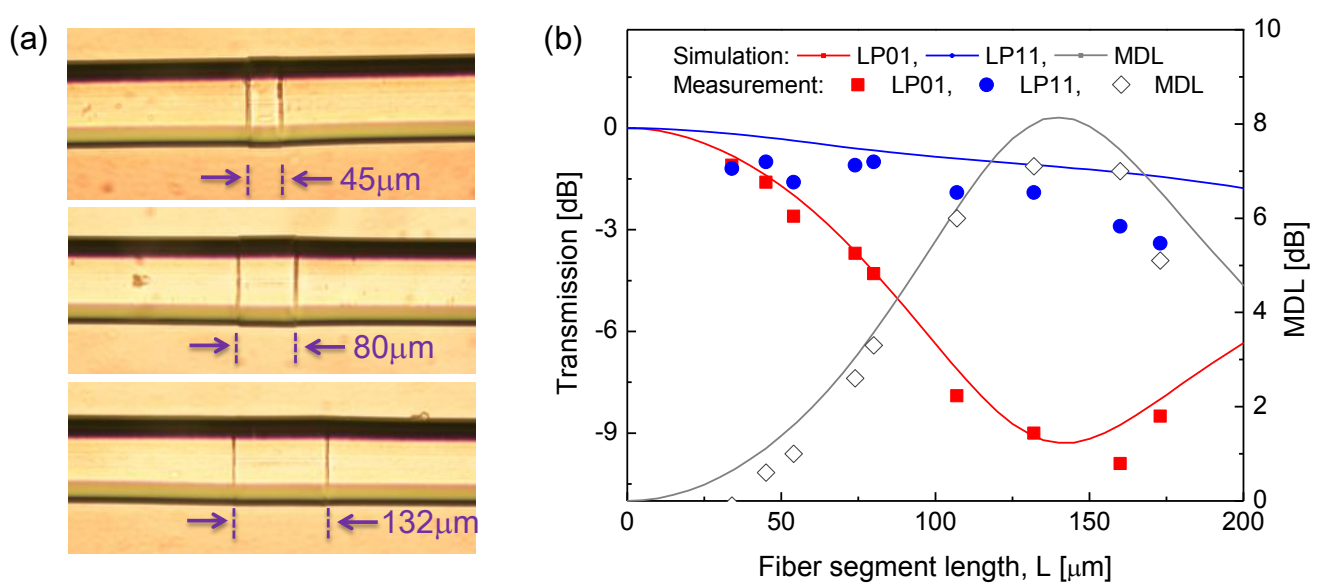

Fig. 4. (a) Microscope images of various lengths of small-core SMF fusion-spliced between two FMFs. (b) The LOM spatial filter response as a function of small-core SMF length.

Fig. 4(a) shows microscope images of the fabricated LOM filters. The lengths of the small-core SMF segments $(d=5 \mu \mathrm{m}, \mathrm{NA}=0.17)$ were $45,80,132 \mu \mathrm{m}$. For various fiber segment lengths, we characterized the mode dependent losses by selectively launching the pure $\mathrm{LP}_{01}$ and $\mathrm{LP}_{11}$ modes using a phase-plate based mode multiplexer. As expected, we can induce LOM filter performance with an 8dB MDL equalization range and a minimal insertion loss $(<2 \mathrm{~dB})$ was achieved for the $\mathrm{LP}_{11}$ in Fig. 4(b), which agrees very well with our simulated results, confirming the viability of the approach.

\section{Conclusion}

We have successfully demonstrated a practical spatial mode selective filter using an all-fiber FMF-SMF-FMF structure. The small-core SMF in the middle creates two MZI optical paths (i.e. a guided mode along the core and a diffracted beam within the cladding) and which can be used to suppress the lower-order modes in a few mode fiber, as confirmed by both theory and experiments. The spatial filter provides excellent lower-order mode filtering performance with an 8dB MDL equalization range and a minimum insertion loss of the higher-order mode of less than $2 \mathrm{~dB}$ in a two mode group fiber. This all-fiber spatial mode filter can provide a cost-effective solution to help mitigate the effect of the MDL and improve the BER performance of MDM systems. It should be possible to extend this concept to fibers supporting a much larger number of modes without incurring significant additional excess loss.

This work was supported by the European Communities $7^{\text {th }}$ Framework Programme under grant agreement 258033 (MODE-GAP).

\section{References}

[1] D. J. Richardson et al., "Space-division multiplexing in optical fibres," Nature Photonics 7, 354-362 (2013).

[2] V.A.J.M.Sleiffer et al., "Field demonstration of mode-division multiplexing upgrade scenarios on commercial networks," Opt. Exp. 21, 31036-31046 (2013).

[3] Y. Jung et al., "Three mode Er3+ ring-doped fiber amplifier for mode-division multiplexed transmission,” Opt. Express 21, 10383-10392 (2013).

[4] I. Weiss et al., "Dynamic mode group equalization filter and variable optical attenuator for few mode fibers," OFC'14, Th4A.8 (2014).

[5] Y. Jung et al., "Ultracompact in-line broadband Mach-Zehnder interferometer using a composite leaky hollow optical fiber waveguide," Opt.

Lett. 33, 2934-2936 (2008). 